\title{
Sequence analysis and characterization of pyruvate kinase from Clonorchis sinensis, a 53.1-kDa homopentamer, implicated immune protective efficacy against clonorchiasis
}

Tingjin Chen ${ }^{1,2,3 \dagger}$, Hongye Jiang ${ }^{1,2,3 \dagger}$, Hengchang Sun ${ }^{1,2,3}$, Zhizhi Xie ${ }^{4}$, Pengli Ren ${ }^{5}$, Lu Zhao ${ }^{1,2,3}$, Huimin Dong ${ }^{1,2,3,4}$, Mengchen Shi ${ }^{1,2,3}$, Zhiyue LV ${ }^{1,2,3}$, Zhongdao Wu ${ }^{1,2,3}$, Xuerong $\mathrm{Li}^{1,2,3}$, Xinbing $\mathrm{Yu}^{1,2,3}$, Yan Huang ${ }^{1,2,3^{*}}$ and Jin $\mathrm{Xu}^{1,2,3^{*}}$

\begin{abstract}
Background: Clonorchis sinensis, the causative agent of clonorchiasis, is classified as one of the most neglected tropical diseases and affects more than 15 million people globally. This hepatobiliary disease is highly associated with cholangiocarcinoma. As key molecules in the infectivity and subsistence of trematodes, glycolytic enzymes have been targets for drug and vaccine development. Clonorchis sinensis pyruvate kinase (CsPK), a crucial glycolytic enzyme, was characterized in this research.

Results: Differences were observed in the sequences and spatial structures of CSPK and PKs from humans, rats, mice and rabbits. CsPK possessed a characteristic active site signature (IKLIAKIENHEGV) and some unique sites but lacked the $\mathrm{N}$-terminal domain. The predicted subunit molecular mass (Mr) of CsPK was $53.1 \mathrm{kDa}$. Recombinant CsPK (rCsPK) was a homopentamer with a Mr. of approximately $290 \mathrm{kDa}$ by both native PAGE and gel filtration chromatography. Significant differences in the protein and mRNA levels of CSPK were observed among four life stages of C. sinensis (egg, adult worm, excysted metacercaria and metacercaria), suggesting that these developmental stages may be associated with diverse energy demands. CsPK was widely distributed in adult worms. Moreover, an intense Th1-biased immune response was persistently elicited in rats immunized with rCsPK. Also, rat anti-rCsPK sera suppressed C. sinensis adult subsistence both in vivo and in vitro.
\end{abstract}

Conclusions: The sequences and spatial structures, molecular mass, and expression profile of CsPK have been characterized. rCSPK was indicated to be a homopentamer. Rat anti-rCSPK sera suppressed C. sinensis adult subsistence both in vivo and in vitro. CSPK is worthy of further study as a promising target for drug and vaccine development.

Keywords: Clonorchis sinensis, Pyruvate kinase, Pentamer, Expression profile, Excretory/secretory products, Immune response, Drug target, Vaccine candidate

\footnotetext{
* Correspondence: huang66@mail.sysu.edu.cn; xujin@mail.sysu.edu.cn

${ }^{\dagger}$ Equal contributors

'Department of Parasitology, Zhongshan School of Medicine, Sun Yat-sen

University, 74 Zhongshan 2nd Road, Guangzhou, Guangdong 510080, China

Full list of author information is available at the end of the article
} 


\section{Background}

Clonorchis sinensis, the causative agent of clonorchiasis, predominantly occurs in East Asia, including China, parts of Russia, South Korea, and northern Vietnam. Globally, it has been estimated that over 200 million people are at risk for clonorchiasis and that more than 15 million persons, of whom 13 million are in China, are infected [1]. Clonorchiasis represents a major tropical disease that is currently neglected [2]. Clonorchis sinensis is considered a group I carcinogen and an important risk factor for cholangiocarcinoma, a fatal malignant tumour of the bile duct that is usually associated with poor prognosis. It has been estimated that as many as 5000 cholangiocarcinoma cases resulting from clonorchiasis may occur yearly for decades to come [2-5]. During long-term infections, the excretory/secretory products (ESPs) of the worms are continuously discharged into the host. Therefore, as molecules that participate in host-parasite interactions, ESPs have been targeted for vaccine and drug development [6-8].

Transcriptomic and genomic data show that the enzymes that participate in the glycolytic pathway and the Krebs cycle are expressed during $C$. sinensis infection $[9,10]$. Exogenous glucose is transported in the musculature of $C$. sinensis adults and is broken down during glycolysis, yielding lactic acid as a major end product and providing metabolic intermediates as well as energy [11, 12]. Glycolytic enzymes are extremely pertinent to the infectivity and subsistence of trematodes, and they have been well characterized as potential targets for drug and vaccine development [6, 13-15].

As a central metabolic regulator in most species, pyruvate kinase (PK, EC: 2.7.1.40, ATP: pyruvate phosphotransferase) catalyses the final ratelimiting reaction in glycolysis, essentially irreversibly transforming phosphoenolpyruvate (PEP) to pyruvate with the formation of one molecule of ATP. Additionally, both the substrates and the products of PK are involved in many biosynthetic pathways as well as in energy metabolism, placing it at a crucial metabolic intersection. In many parasites, including Leishmania mexicana, Trypanosoma brucei, Toxoplasma gondii, Plasmodium falciparum and Schistosoma mansoni, PK is highly regulated, indicating that it may play crucial regulatory roles in glycolysis in these parasites [15-19]. PK is considered a compelling therapeutic target for malignant tumours as well as for human pathogens such as apicomplexans [20]. Nearly all PKs exist as homotetramers [18, 21].

At present, research on the crucial glycolytic enzymes of $C$. sinensis is even more limited than research on the glycolytic enzymes of protozoa. Although PKs from L. mexicana $[15,22,23]$, T. gondii $[17,20,24]$, T. brucei [16], Trypanosoma cruzi [22], P. falciparum [18], Eimeria tenella [25], and Entamoeba histolytica [26] have been characterized in detail, the characteristics of $C$. sinensis PK $(C s \mathrm{PK})$ as a potential target for drug and vaccine development are unknown.
CsPK is a $53.1-\mathrm{kDa}$ homopentamer that possesses a characteristic active site signature (IKLIAKIENHEGV) as well as some unique sites but lacks the characteristic PK N-terminal domain. The mRNA levels, protein levels and tissue distribution of $C s \mathrm{PK}$ at various life stages of $C$. sinensis were characterized. A T helper cell (Th) 1-biased immune response was induced in $\mathrm{rCs}$ PK-immunized rats. Rat anti-rCsPK sera suppressed $C$. sinensis adult subsistence in vivo and in vitro. This research indicates that CsPK may be a promising candidate for the development of drugs and vaccines for clonorchiasis.

\section{Methods}

\section{Sequence analysis}

By using nucleotide sequence of PK gene (identify the query sequence), since they had used BLASTx algorithm (http://www.ncbi.nlm.nih.gov/), amino acid sequences of PK were retrieved from GenBank including that of $C$. sinensis (GAA54498.1). The physicochemical parameters and conserved domains of $C s \mathrm{PK}$ were analysed using bioinformatics tools (ProtParam, InterProScan) in ExPASy (http://www.expasy.org/). The linear B cell epitopes were analysed with BepiPred (http://tools.immuneepitope.org/ main/). The putative tertiary structure of CsPK was simulated by comparative modelling using SWISS-MODEL (https://swissmodel.expasy.org/); the resulting structure was viewed using Swiss-Pdb Viewer and further evaluated using the Q-MEAN server. A phylogenetic tree of the PKs was constructed using the neighbour-joining method in MEGA version 5. Tests of phylogeny were conducted using the Bootstrap method with 1000 replications. Protein weight matrix was performed using Gonnet. The amino acid substitution model was constructed using the p-distance method.

\section{Expression and purification of recombinant CsPK (rCsPK)}

The open reading frame (ORF) of CsPK was amplified by the polymerase chain reaction (PCR) from the total cDNA of adult $C$. sinensis using specific primers (F: 5'GCA CGG ATC CAT GCT ACA GAA GAT GA-3', R: 5'-ATA GTC GAC AAA GCG GCT GAA GCT C-3'). The PCR products were purified and cloned into the prokaryotic expression vector pET-28a(+) (Novagen, Darmstadt, Germany). The recombinant plasmids were confirmed by digestion using the corresponding restriction enzymes and identified by DNA sequencing.

The recombinant plasmids were transformed into Escherichia coli BL21 (DE3) (Promega, Madison, USA). After being induced with $1 \mathrm{mM}$ isopropyl- $\beta$-D-thiogalactopyranoside (Sigma-Aldrich, St. Louis, USA) at $37{ }^{\circ} \mathrm{C}$ for $6 \mathrm{~h}$, the transformed cells were collected by centrifugation and sonicated on ice. $\mathrm{rCs} \mathrm{PK}$ was overexpressed as inclusion bodies. The sediment containing the His-tagged recombinant protein $(\mathrm{rCs} \mathrm{PK})$ was washed with washing buffer (50 mM Tris- $\mathrm{HCl}, 50 \mathrm{mM} \mathrm{NaCl}, 1 \%$ 
Triton X-100, 1 mM EDTA, $2 \mathrm{M}$ urea, $\mathrm{pH}$ 8.0) and dissolved in dissolving buffer $(50 \mathrm{mM}$ Tris- $\mathrm{HCl}, 50 \mathrm{mM} \mathrm{NaCl}, 1 \%$ Triton X-100, 1 mM EDTA, $6 \mathrm{M}$ urea, $\mathrm{pH}$ 8.0) followed by centrifugation. The inclusion bodies dissolved readily in $6 \mathrm{M}$ urea. The supernatant was applied to a $\mathrm{Ni}^{2+}$-NTA affinity column and eluted with a gradient of 5-300 $\mathrm{mM}$ imidazole containing $6 \mathrm{M}$ urea. The protein eluted at $80-300 \mathrm{mM}$ imidazole with high purity. The purified protein was renatured by sequential dialysis against solutions containing decreasing concentrations of urea (6 M to $0 \mathrm{M})$. Finally, the renatured protein was dialysed against phosphate-buffered saline (PBS).

The purified protein was subjected to electrophoresis on a $12 \%$ sodium dodecyl sulphate-polyacrylamide gel (SDS-PAGE) and identified by mass spectrometry (MS). Peptide mass spectra were performed on an ABI 4800 Proteomics Analyzer MALDI-TOF/TOF (Applied Biosystems, Md., USA). Both the MS and tandem mass spectrometry (MS/MS) data were interpreted and processed using the GPS Explorer software (version 3.6; Applied Biosystems, Md., USA) to match the protein name.

\section{Preparation of parasites, total worm extracts, CsESPs and antisera against $\mathrm{rCsPK} / \mathrm{CsESPs}$}

Clonorchis sinensis metacercariae were collected from experimentally infected freshwater Ctenopharyngodon idellus fish at our laboratory's pool [27]. Viability and integrity of metacercariae were assessed under a microscope. $0.001 \%$ trypsin (Promega) in physiological saline was employed as an excystation stimulus in vitro. After activation, some encysted metacercariae was immediately collected in RIPA lysis buffer containing $1 \mathrm{mM}$ phenylmethanesulfonyl fluoride (PMSF; Bioteke, Beijing, China) for extraction of total protein. Moreover, some encysted metacercariae were immediately collected for extraction of total RNA using TRIzol (Invitrogen, California, USA). Each SD rat was intragastrically infected with 50 metacercariae. At week 8 post-infection, the rats were sacrificed, and adult $C$. sinensis were recovered from their bile ducts.

Total worm extracts, CsESPs and mouse anti-CsESPs sera were obtained as described previously [6]. Briefly, living adult $C$. sinensis were cultured in DMEM (Gibco) under $5 \% \mathrm{CO}_{2}$ at $37{ }^{\circ} \mathrm{C}$. CsESPs were collected from the culture medium, treated with $1 \mathrm{mM}$ PMSF, centrifuged and dialyzed in PBS. Living adult $C$. sinensis were crushed to prepare total worm extracts, stored at $-80^{\circ} \mathrm{C}$. Rats and mice were subcutaneously immunized with 200/50 $\mu$ g purified $\mathrm{rCs}$ PK emulsified with an equivalent volume of complete Freund's adjuvant (Sigma-Aldrich) followed by 2 boosters of $\mathrm{rCsPK}(100 / 25 \mu \mathrm{g})$ emulsified with an equivalent volume of incomplete Freund's adjuvant (Sigma-Aldrich) at 2-week intervals. Preimmune sera were harvested before the first immunization.
Immune sera were harvested at 2-week intervals 2 to 24 weeks after immunization.

\section{Determination of the apparent molecular mass (Mr) of rCsPK}

Purified $\mathrm{rCsPK}$ was subjected to native PAGE on $8 \%$ gels. The Mr. of $\mathrm{rCs} \mathrm{PK}$ was confirmed by determining the relationship between the log Mr. $(\mathrm{kDa})$ of the standard marker proteins (Sigma-Aldrich) and their elution volumes (Ve) on AKTA FPLC using Sepharose 12 10/300 GL gel filtration chromatography (GFC) (GE Healthcare, Pittsburgh, USA) [28]. The GFC column was run at a flow rate of $0.8 \mathrm{ml} / \mathrm{min}$ in $50 \mathrm{mM}$ Tris- $\mathrm{HCl}$ containing $100 \mathrm{mM} \mathrm{KCl}(\mathrm{pH} \mathrm{7.5).}$ Based on the deduced equation, the Mr. of $\mathrm{rCsPK}$ was derived using the obtained $\mathrm{Ve}$ of $\mathrm{rCs} \mathrm{PK}$.

\section{Western blotting}

Purified rCsPK $(2.5 \mu \mathrm{g})$, CsESPs $(25 \mu \mathrm{g})$ and total worm extract $(25 \mu \mathrm{g})$ were separated by $12 \%$ SDS-PAGE and electrotransferred to a PVDF membrane in a Trans-Blot transfer cell (Bio-Rad, Hercules, USA) at $100 \mathrm{~V}$ for $1 \mathrm{~h}$. The PVDF membranes were blocked with $5 \%$ skim milk in PBS ( $\mathrm{pH} 7.4$ ) at $4{ }^{\circ} \mathrm{C}$ overnight and then incubated with a mouse His-tagged monoclonal antibody (1:2000 dilution, Novagen, Darmstadt, Germany), mouse anti$\mathrm{rCsPK}$ serum (1:2000 dilution), serum of mice infected with $C$. sinensis (1:2000 dilution), mouse anti-CsESP serum (1:2000 dilution) or preimmune mouse serum (1:2000 dilution) at RT for $2 \mathrm{~h}$. After 3 washes in PBS, the membranes were incubated with HRP-conjugated goat anti-mouse IgG (1:2000 dilution) at RT for $1 \mathrm{~h}$. After washing the membranes 5 times, detection was performed using chemiluminescence.

\section{CsPK mRNA and protein levels at various life stages of $C$. sinensis}

Total RNAs were isolated from the egg, adult, excysted metacercaria and metacercaria of $C$. sinensis using TRIzol (Invitrogen). Clonorchis sinensis $\beta$-actin (accession No. EU109284) was used as an internal control, which was amplified with specific primers 5 '-ACC GTG AGA AGA TGA CGC AGA-3' and 5'-GCC AAG TCC AAA CGA AGA ATT-3'. Real-time PCR was performed using the SYBR Premix Ex Taq Kit (Takara, Shiga, Japan) in the iQ5 Real-Time PCR Detection System (Bio-Rad, Hercules, USA) using specific primers 5 '-AAG ATA AGG CAG ATT TAC GCT-3' and 5'-CTG GGA TTT CAA TAC CAAGAT- 3 '. The PCR program was $95{ }^{\circ} \mathrm{C}$ for $5 \mathrm{~min}$ followed by 40 cycles of $95{ }^{\circ} \mathrm{C}$ for $10 \mathrm{~s}$ and $56{ }^{\circ} \mathrm{C}$ for $30 \mathrm{~s}$. Melting curves were obtained by heating the samples to $95^{\circ} \mathrm{C}$ for $30 \mathrm{~s}$ and $60^{\circ} \mathrm{C}$ for $15 \mathrm{~s}$ followed by an increase in temperature to $95^{\circ} \mathrm{C}$. Semiquantitative analysis was conducted according to the $2^{-\Delta \Delta C T}$ method $[29,30]$. 
Western blotting was used to study the levels of CsPK protein present at the above 4 life stages of $C$. sinensis. Parasites from those stages were suspended in RIPA lysis buffer containing $1 \mathrm{mM}$ PMSF. A supernatant was obtained by centrifugation at $10,000 \times g$ for $20 \mathrm{~min}$ at $4{ }^{\circ} \mathrm{C}$. Total protein $(40 \mu \mathrm{g})$ of each stage was subjected to $12 \%$ SDS-PAGE and blotted onto PVDF membrane. The blots were incubated with sera from mice immunized with $\mathrm{rCs}$ PK (1:200 dilution) or with mouse preimmune sera (1:200 dilution) followed by incubation with HRPconjugated goat anti-mouse IgG (1:2000 dilution). Detection was performed with chemiluminescence. Relative protein levels were quantitated using Tanon Gis software (Tanon 4100, Shanghai, China).

\section{Immunolocalization of CsPK in C. sinensis}

Adults and metacercariae of $C$. sinensis were fixed in formalin, embedded in paraffin wax and cut into $5-\mu \mathrm{m}$-thick sections. After successive deparaffinization with xylene and hydration in a graded ethanol series, the sections were blocked with normal goat serum at RT for $2 \mathrm{~h}$. The sections were then incubated with mouse anti-rCsPK serum (1:100 dilution) in a humidified chamber overnight at $4{ }^{\circ} \mathrm{C}$. Mouse preimmune serum diluted at the same ratio was used as a negative control. The sections were washed 3 times with PBS containing 0.05\% Tween-20 (PBS-T, pH 7.4) and 2 times in PBS followed by incubation with Cy3-conjugated goat anti-mouse IgG (1:400 dilution, Molecular Probes, USA) in the dark at RT for $1 \mathrm{~h}$. BSA $(0.1 \%)$ in PBS was used as the dilution buffer. The sections were imaged using a fluorescence microscope (Leica, Wetzlar, Germany).

\section{Enzyme-linked immunosorbent assay (ELISA) of antibody titres and isotypes of IgG elicited by $\mathrm{rCsPK}$}

Microplates were incubated overnight at $4{ }^{\circ} \mathrm{C}$ with $2.5 \mu \mathrm{g}$ /well-purified $\mathrm{rCs} \mathrm{PK}$ in coating buffer $(0.1 \mathrm{M}$ carbonate-bicarbonate, $\mathrm{pH}$ 9.6). The plates were then blocked with $5 \%$ skim milk in PBS-T at $37{ }^{\circ} \mathrm{C}$ for $2 \mathrm{~h}$. The protein bound to the wells was incubated with serial dilutions of the immune sera (obtained at week 6 following the first immunization) elicited by $\mathrm{rCs} \mathrm{PK}$ followed by washing. Serum from rats or mice immunized with PBS was used as a negative control. HRP-conjugated goat anti-host specific IgG (1:20,000 dilution in $0.1 \%$ BSA PBS-T, Proteintech, Rosemont, USA) was used as the secondary antibody. After incubation for $1 \mathrm{~h}$ and 3 washes in PBS-T, $100 \mu$ l of substrate solution (TMB, BD Biosciences, San Diego, USA) was added to each well followed by incubation for $12 \mathrm{~min}$ in the dark. After the addition of $2 \mathrm{M} \mathrm{H}_{2} \mathrm{SO}_{4}$ to terminate the reaction, the absorbance at $450 \mathrm{~nm}$ was measured. The levels of total IgG and IgG isotypes in sera obtained at various time points $(2,4,6,8,10,12,14,16,18,20,22$ and 24 weeks after the first immunization) were also measured. The primary antibodies were diluted 1:400. HRP-conjugated goat anti-rat IgG (1:20,000 dilution) and IgG1/IgG2a (1:10,000 dilution, Bethyl, TX, USA) were used as secondary antibodies.

\section{Immune protection conferred by $\mathrm{rCsPK}$}

Thirty-two 6-week-old SD rats were randomly separated into 4 groups: an adjuvant group, an infection group, a rCsPK group, and a PBS group ( $n=8$ for each group). The SD rats in the $\mathrm{rCs} \mathrm{PK}$ and PBS groups received subcutaneous injections of either $200 \mu \mathrm{g}$ rCsPK or an equal volume of PBS, respectively, emulsified with complete Freund's adjuvant. Two boosters of $100 \mu \mathrm{g}$ rCsPK or an equal volume of PBS emulsified with incomplete Freund's adjuvant were given at 2 -week intervals. The SD rats in the adjuvant group received subcutaneous injections of an equal volume of adjuvant. The SD rats in the infection group received no pretreatment.

After measurement of the animals' antibody titres at 6 weeks after immunization, all rats were challenged by oral administration of 80 live $C$. sinensis metacercariae. The eggs per gram faeces (EPG) were calculated at week 6 post-infection. For worm burden evaluation, the rats were sacrificed 8 weeks post-infection, and adult $C$. sinensis were recovered from their livers. The EPG and worm burden were calculated blindly. The reduction in the parasite burden was calculated as follows: egg reduction rate $(\%)=[$ (average EPG of control group - average EPG of experimental group) / average EPG of control group $] \times 100 \%$; worm reduction rate $(\%)=[$ (average worm burden of control group - average worm burden of experimental group) / average worm burden of control group] $\times 100 \%$ [31] .

\section{Culture of $C$. sinensis adults in the presence of rat anti- rCsPK sera}

Clonorchis sinensis adults were recovered from infected rats, washed 4 times with sterilized PBS containing 1\% antibiotics (streptomycin $100 \mathrm{U} / \mathrm{ml}$ and penicillin $100 \mu \mathrm{g} / \mathrm{ml}$ ) and transferred to 12 -well plates at 20 adults per well in $2 \mathrm{ml} /$ well of low-glucose DMEM containing $1 \%$ antibiotics. Serum from rats that had been immunized with $\mathrm{rCs} \mathrm{PK}$ or preimmune serum from the same animals was mixed with the medium at dilutions of 1:40-1:160. Low-glucose DMEM was used as the blank control. At 1-10, 15, 18, 20, 22, 24, 26 and 28 days of incubation, the adult worms were counted and monitored for 5 min using a microscope (Leica). Adults that failed to display pumping and muscle contraction after 5 continuous mechanical stimuli were classified as dead [32].

\section{Statistics and software}

All experiments were performed at least 3 times. SPSS software (version 17.0; SPSS, Inc., IL, USA) was 
employed for statistical analysis. Differences in IgG isotypes and immune protection between the groups were analysed using Student's $t$-test. The survival rates of cultured adults were analysed using the Kaplan-Meier method, and differences between the groups were determined using the log-rank (Mantel-Cox) test. The results of the analyses are expressed as the mean \pm standard deviation; significance was set at a $P$-value $<0.05$.

\section{Results}

\section{Sequence analysis and putative spatial structure of CsPK}

The ORF of CsPK is $1458 \mathrm{bp}$ in length and encodes 485 amino acids (aa). In its protein sequence, CsPK shows the identities to the PK of Opisthorchis viverrini (99\%), followed Schistosoma japonicum (80\%), Brugia malayi (59\%), Hymenolepis microstoma (39\%) and Echinococcus granulosus (33\%). The protein sequence included the characteristic active site signature (IKLIAKIENHEGV) (Fig. 1). This sequence, which occurs between amino acid residues 203 and 215 of $C s \mathrm{PK}$, contains a lysine (K) residue that forms part of the catalytic site and that appears to be responsible for interconverting enolpyruvate and pyruvate. L205 was unique to $C s \mathrm{PK}$, replacing the usual isoleucine (I). A207 of CsPK replaced the usual serine $(S)$ found in the rat, mouse and human enzymes (Additional file 1: Figure S1). The consensus regions for the binding of PEP, ADP, and monovalent as well as divalent cations were well conserved.

In the sequence alignment, the 4 three-dimensional (3D) domains described for PK are shown. The N-terminal domain, which only appears in eukaryotic PKs, is very long in S. japonicum, T. gondii and E. tenella but absent in C. sinensis. Domains A and B, which contain the catalytic sites, are well conserved. In contrast, domain $\mathrm{C}$, which contains the effector sites and is localized at the C-terminal end, is diverse [25]. CsPK contains 22 predicted linear B cell epitopes and lacks a transmembrane region and a signal peptide. CsPK was predicted to be expressed in the cytosol [24].

The 3D structure of CsPK was simulated using SWISSMODEL based on the molecular model of truncated TgPK1 (an N-terminal truncated version, Protein Data Bank PDB: 3GG8 [20]), which exhibited $44.67 \%$ identity to CsPK. The GMQE score of 0.74 and the Q-MEAN4 of -0.83 supported the quality of the model. CsPK consisted only of domain A (blue), domain B (red) and domain C (green). The catalytic sites appear at the interface of domains A and B (Additional file 1: Figure S1). It is notable that the K454-S459 region and the G413-D423 loop, which corresponds to the mobile loop in yeast PK [33], are components of the allosteric binding site [20].

In human PK-M2 (PDB: 3BJF [34]) and CsPK, the F16BP binding sites, which interact with the phosphate moieties, are also conserved with different conformations. The ribbon drawing shows a superposition between domain $\mathrm{A}$ of $C s \mathrm{PK}$ with the closed rabbit PK-M1 in complex with ATP and the inhibitor oxalate (PDB: 1A49 [35]). The significant residues of the two proteins are highly conserved and show diverse conformations.

In the active site of CsPK and in that of LmPYK-suramin (PDB: 3PP7 [22]) complexed with glycerol and suramin (an inhibitor of $T$. brucei glycolytic enzymes), the significant residues are highly conserved and exhibit the same conformation.

In the phylogenetic tree of PKs (Fig. 2), CsPK grouped very closely with the PKs of trematodes. $C s \mathrm{PK}$ was closely related to PKs of vertebrates, followed by those of nematodes and cestodes, whereas it was distantly related to the PKs of protozoa. Phylogenetic, sequence and structural analysis revealed that CsPK (T52 and E56) is related to the group of monovalent cation-dependent isozymes (type I) [24]. In contrast, CsPKII (GAA58090.1, 245 aa) clusters with $E$. coli PKII (AAA24473.1), along with three isozymes from the protozoans $T$. gondii (KFH06835.1), P. falciparum (AAN35560) and T. parva (XP_764703.1). These results suggest a different evolutionary origin of the two isozymes in C. sinensis.

\section{Expression and apparent Mr. of $\mathrm{rCsPK}$}

The putative theoretical isoelectric point of $C s \mathrm{PK}$ is 6.69, and its predicted subunit Mr. is $53.1 \mathrm{kDa}$. Purified $\mathrm{rCs} \mathrm{PK}$ containing a $6 \times$ His-tag displayed a single band of approximately $58.0 \mathrm{kDa}$, consistent with the calculated Mr. (Fig. 3a, Lane 5). The peptides obtained from the purified protein, which was demonstrated by MS analysis, were matched to those of $C s \mathrm{PK}$ at a protein coverage of $20 \%$ (data not shown).

Native PAGE and molecular sieve chromatography were used to determine the protein's multimeric status. In native PAGE (Fig. 3b), the mobility of $\mathrm{rCsPK}$ was between that of ferritin $(440 \mathrm{kDa})$ and that of catalase $(232 \mathrm{kDa})$. According to the equation describing the electrophoretic profile, the Mr. of $\mathrm{rCs} \mathrm{PK}$ was approximately $290 \mathrm{kDa}$.In GFC, rCsPK eluted as a single peak between $\beta$-amylase $(200 \mathrm{kDa})$ and ferritin $(440 \mathrm{kDa})$ (Additional file 2: Figure S2).

\section{Western blotting}

rCsPK was recognized by a mouse His-tagged monoclonal antibody, mouse anti-rCsPK sera, sera of mice infected with $C$. sinensis and mouse anti-CsESPs sera; in all cases, a cross-reactive band of approximately $58.0 \mathrm{kDa}$ (including the Mr. of the His-tag) was noted. This immunoreactive band did not appear on blots that had been probed with naive mouse sera. Additionally, CsESPs and total worm extracts probed with mouse anti-rCsPK sera, but not with preimmune sera, showed an immunoreactive band of approximately $53.1 \mathrm{kDa}$ (Fig. 4). 


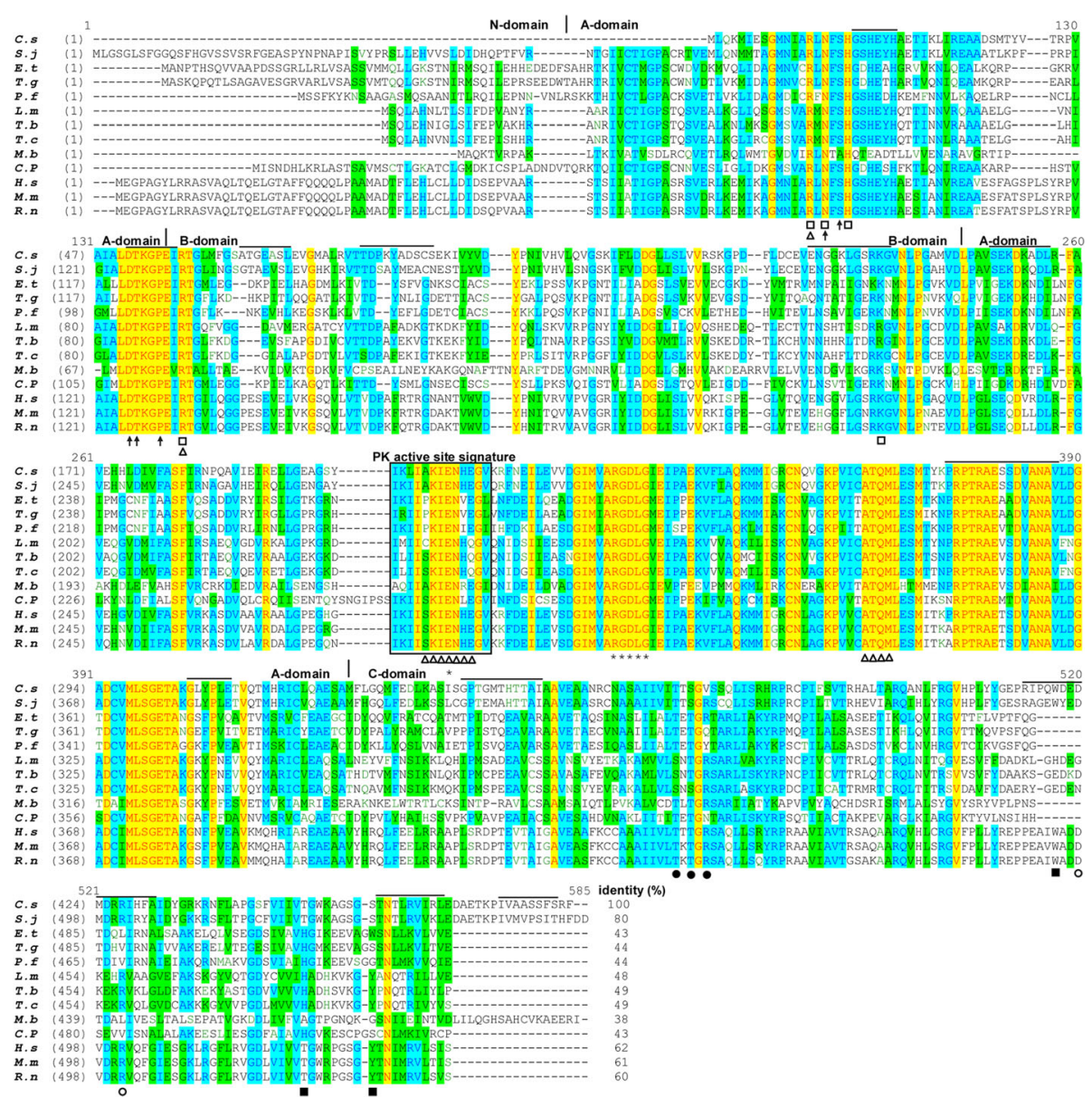

Fig. 1 Sequence analysis of CsPK. An alignment of the protein sequence of CsPK with those of PKs from other organisms is shown. Clonorchis sinensis (C.S, GAA54498.1), Schistosoma japonicum (S.j, AAW27129.1), Eimeria tenella (E.t, AAC02529.1), Toxoplasma gondii (T.g, BAB47171.1), Plasmodium falciparum (P.f, CAD50538.1), Leishmania mexicana (L.m, CAA52898.2), Trypanosoma brucei (T.b, P30615.1), Trypanosoma cruzi (T.C, EKG02834.1), Mastigamoeba balamuthi (M.b, AAK94944.1), Cryptosporidium parvum (C.p. 4DRS_A), Homo sapiens (H.s, AAA60104.1), Mus musculus (M.m, NP_001093249.1), and Rattus norvegicus (R.n, AAA41880.1). The 3D-domains (N/A/B/C) are marked with vertical lines. The 22 predicted B cell linear epitopes with more trustworthiness are indicated by the black lines above the alignment. The rectangle indicates the PK active site signature. The triangles and open squares indicate the PEP and ADP binding sites, respectively. The binding sites of the sugar, 1-phosphate and 6-phosphate moieties of the allosteric activator fructose-1,6bisphosphate (F16BP) are indicated by closed squares, open circles and closed circles, respectively. The black arrows and asterisks indicate monovalent cation and divalent cation binding sites, respectively

mRNA and protein levels and immunolocalization of CsPK at the life stages of $C$. sinensis

The mRNA levels of CsPK were measured at the adult, egg, excysted metacercaria, and metacercaria stages of $C$. sinensis (Fig. 5a). Significant differences were observed in the mRNA levels of $C s \mathrm{PK}$ at the above 4 life stages $(P<0.01)$. The mRNA level of CsPK in the egg was higher than that in the adult (56.79-fold, $\left.t_{(2)}=17.392, P=0.003\right)$, metacercaria (9.72-fold, $\left.t_{(2.02)}=15.844, P=0.004\right)$ and excysted metacercaria (5.97-fold, $t_{(4)}=14.477, P<0.001$ ).

A specific polypeptide of molecular weight approximately $53.1 \mathrm{kDa}$ was detected with mouse anti-rCsPK sera by Western blotting analysis. The protein level of CsPK was highest in the egg, followed by excysted metacercaria, metacercaria and adult (Fig. 5b, c). No corresponding band was detected when preimmune mouse serum was used (data not shown).

In sections of adult worms (Fig. 6), strong fluorescence due to $C s \mathrm{PK}$ was observed in the oral sucker, ventral sucker, pharynx, genital pore, vitellarium, tegument, intestine, seminal receptacle, testicle, ovary, uterus and in eggs within the uterus. Moreover, strong fluorescence was apparent on the tegument, oral sucker, ventral sucker, and vitellarium, in metacercariae. No specific fluorescence was observed in negative controls incubated with preimmune mouse serum.

\section{IgG isotypes and immune protective efficacy elicited by rCsPK}

The titre of anti-rCsPK IgG in rats and mice was as high as $1: 409,600$ to $1: 204,800$ at week 6 post-immunization, 


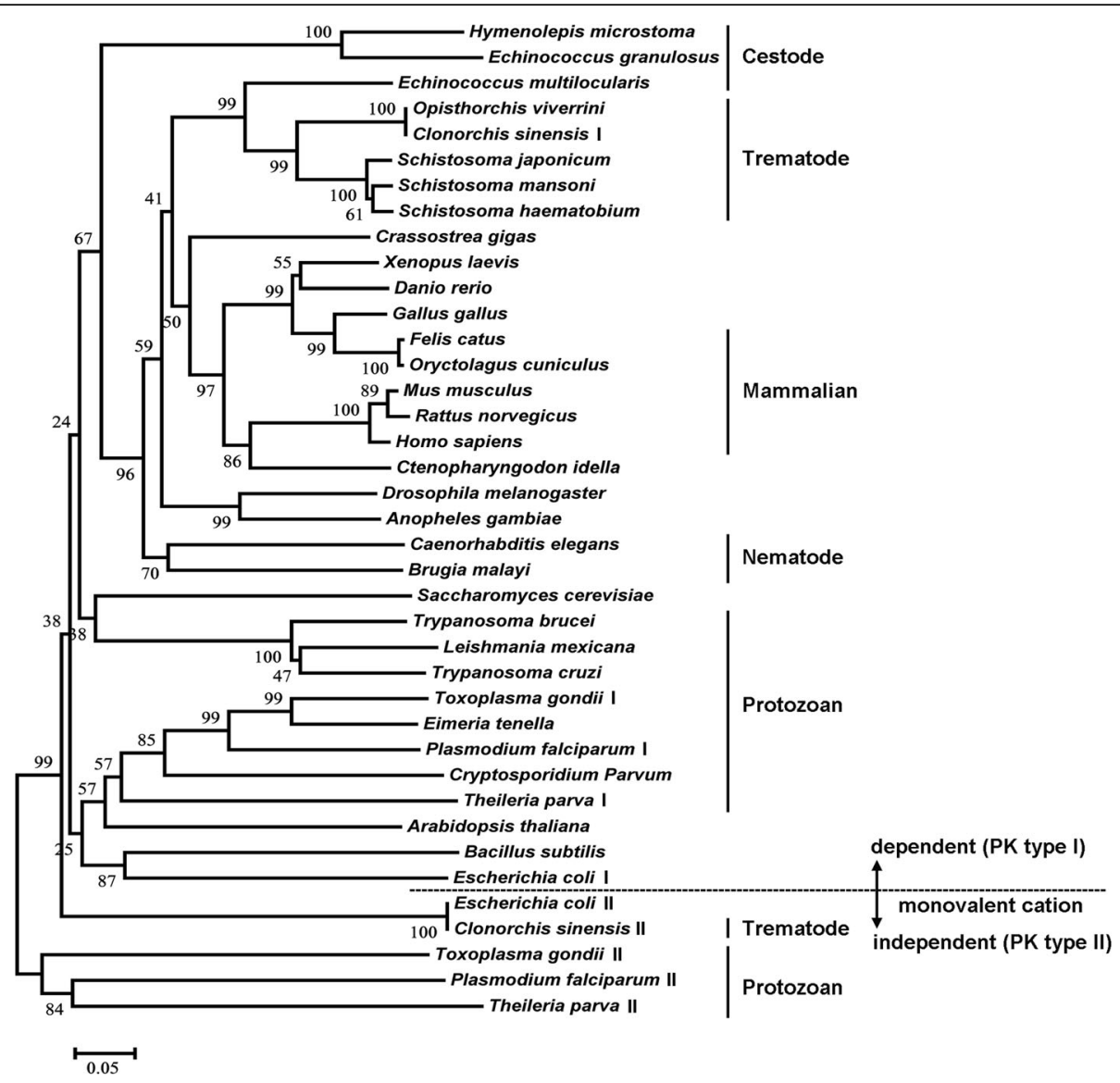

Fig. 2 Neighbour-joining phylogenetic tree of PKs. The bootstrap values are displayed at the branching point (test of phylogeny by the bootstrap method with 1000 replications). The bar indicates the substitution by p-distance method. The protein sequences were obtained from GenBank and DDBJ. The sequences are as follows: Escherichia coli (AAA24392.1, AAA24473.1), Bacillus subtilis (P80885.2), Arabidopsis thaliana (BAB10461.1), Cryptosporidium parvum (4DRS_A), Plasmodium falciparum (CAD50538.1, AAN35560), Eimeria tenella (AAC02529.1), Toxoplasma gondii (BAB47171.1, KFH06835.1), Trypanosoma brucei (P30615.1), Trypanosoma cruzi (EKG02834.1), Leishmania mexicana (CAA52898.2), Theileria parva (XP_764242.1, XP_764703.1), Saccharomyces cerevisiae (CAA24631.1), Brugia malayi (XP_001898626.1), Hymenolepis microstoma (CDS33796.1), Echinococcus granulosus (CDS23463.1), Echinococcus multilocularis (CDS43052.1), Caenorhabditis elegans (CAA93424.2), Anopheles gambiae (EAA10555.6), Drosophila melanogaster (AAC16244.1), Crassostrea gigas (CAJ28914.1), Clonorchis sinensis (GAA54498.1, GAA58090.1), Opisthorchis viverrini (KER20867.1), Schistosoma japonicum (AAW27129.1), Schistosoma haematobium (KGB40466.1), Schistosoma mansoni (CCD76479.1), Danio rerio (NP_955365.1), Xenopus laevis (NP_001084341.1), Gallus gallus (NP_990800.1), Felis catus (P11979.2), Homo sapiens (AAA60104.1), Mus musculus (NP_001093249.1), Rattus norvegicus (AAA41880.1), Ctenopharyngodon idella (AFY98078.1)

indicating that the protein is strongly immunogenic (Fig. 7a, b). In rat anti-rCsPK sera, IgG2a and IgG1 levels increased at week 2 and reached peak values at 6 and 8 weeks. IgG1 levels decreased markedly after 16 weeks, whereas IgG2a levels were maintained at a high level until 24 weeks. Between 2 and 24 weeks postimmunization, the level of IgG2a was significantly higher than that of IgG1 (Fig. 7c).

The EPG values of the PBS group, the adjuvant group, the infection group, and the $\mathrm{rCsPK}$ group were $3983.3 \pm 386.7 ; 4075.0 \pm 473.0 ; 3895.8 \pm 424.1$; and $1716.7 \pm 230.3$, respectively. The numbers of worms recovered from the above 4 groups were $25.1 \pm 4.8$; $24.8 \pm 5.3 ; 26.1 \pm 5.1$; and $11.2 \pm 2.5$, respectively (Table 1). The EPG and worm burden showed a marked decrease in the $\mathrm{rCs} \mathrm{PK}$ group compared with the control groups $\left(t_{(11.410)}=14.245, P<0.001 ; t_{(14)}=7.299\right.$, $P<0.001)$. The egg reduction rate and worm reduction rates were $56.90 \%$ and $55.22 \%$ (compared with the PBS group), respectively. No significant difference in EPG or worm burden was observed among the PBS, adjuvant, and infection groups.

\section{Rat anti-rCsPK sera affects $C$. sinensis adult subsistence in vitro}

The median survival times of $C$. sinensis adults in the blank control group, in preimmune sera groups (with $1: 40,1: 80$ or $1: 160$ dilution), and in anti-rCsPK sera groups (with 1:40, 1:80 or 1:160 dilution) were $15,8,8$, 9, 2, 3 and 4 days, respectively (Fig. 8). No significant difference in survival rate was observed among preimmune sera groups with different dilution $(P>0.05)$. 


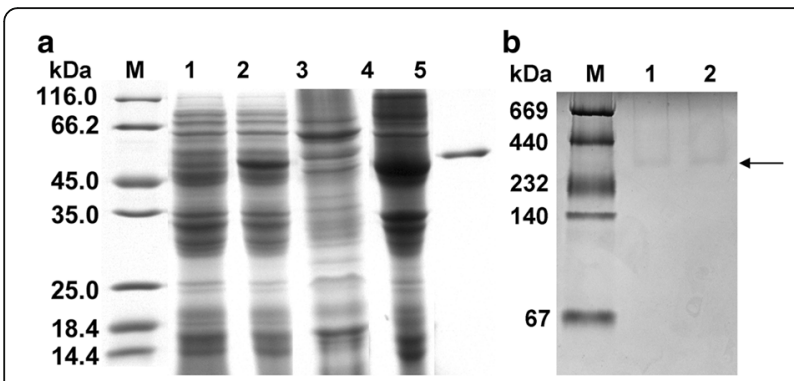

Fig. 3 Expression and apparent Mr. of rCSPK. Proteins were visualized by Coomassie Blue staining. Lane M contains protein molecular weight markers. a Expression and purification of rCSPK. Lysate of E. coli transformed with pET-28a(+)-CSPK without induction (Lane 1) and with induction (Lane 2); supernatant (Lane 3) and precipitate (Lane 4) of lysate of E. coli with pET-28a(+)-CsPK with induction; and purified rCsPK (Lane 5). b In Lane M, the protein bands with known Mr. (in descending order) are thyroglobulin (669 kDa), ferritin (440 kDa), catalase (232 kDa), lactate dehydrogenase (140 kDa), and BSA (67 kDa). Lane 1, Freshly purified rCsPK; Lane 2, rCsPK stored for 4 weeks at $-80^{\circ} \mathrm{C}$ with 4 cycles of freezing and thawing

There were dominant differences of survival rates among all other groups $(P<0.01)$.

\section{Discussion}

In the present research, we investigated and compared the sequence and spatial structure of $C s \mathrm{PK}$ with those of PKs from humans, rats, mice, and rabbits, all of which are definitive hosts of $C$. sinensis. CsPK is a $53.1-\mathrm{kDa}$ homopentamer $\mathrm{PK}$ that lacks the characteristic N-terminal domain but possesses a characteristic active site signature (IKLIAKIENHEGV) and some unique sites. The mRNA levels, protein levels and immunolocalization of $C s \mathrm{PK}$ at various life stages of $C$. sinensis were characterized. $C s \mathrm{PK}$ was a component of CsESPs. Also, a Th1-biased immune response was elicited in rats immunized with $\mathrm{rCs} \mathrm{PK}$. Furthermore, rat anti-rCsPK sera suppressed $C$. sinensis adult subsistence both in vivo and in vitro.

The protein sequence and spatial structure analysis revealed that $C s \mathrm{PK}$ possesses the typical characteristics of a PK (Fig. 1, Additional file 1: Figure S1). To our knowledge, a homopentamer PK from any organism has not previously been reported. The peptides of $\mathrm{rCs} \mathrm{PK}$ were analysed by MS analysis (data not shown). Based on SDS-PAGE, the Mr. of $\mathrm{rCsPK}$ was approximately $58 \mathrm{kDa}$ (including a His-tag) (Fig. 3b). A band of approximately $58 \mathrm{kDa}$ in $\mathrm{rCs} \mathrm{PK}$ reacted with a mouse His-tagged monoclonal antibody (Fig. 4), whereas the Mr. of rCsPK was approximately $290 \mathrm{kDa}$, as confirmed by native PAGE and GFC (Fig. 3, Additional file 2: Figure S2). The above results indicate that $C s \mathrm{PK}$ is a homopentamer, whereas almost all PKs from other species are homotetrameric enzymes [18, 21].

The X-ray crystallographic structures of some PKs of diverse organisms (such as E. coli, L. mexicana, yeast, rabbit muscle, human, and cat) are highly similar with respect to ordinary $\mathrm{PK}$ topology [23, 34-39]. PKs are homotetrameric enzymes consisting of identical subunits of approximately $50-60 \mathrm{kDa}$; each subunit consists of 3 to 4 domains: $\mathrm{N}$ (a variable $\mathrm{N}$-terminal domain), $\mathrm{A}, \mathrm{B}$, and $C[23,40]$. The $\mathrm{N}$-terminal helical domain is only present in eukaryotic PKs and can be removed from human erythrocyte PK without affecting the enzyme's activity or stability [39]. The N-terminal domain is very long in $S$. japonicum but was absent in $C$. sinensis.

In each subunit, the catalytic sites lie at the interface of domains $\mathrm{A}$ and $\mathrm{B}$, and the allosteric sites are located in domain C (Fig. 1, Additional file 1: Figure S1) [20, 35, 37]. Multiple alignment of the protein sequences of PKs from parasitic species with those of human, rat, and mouse PKs (Fig. 1) showed that the known binding sites of PEP, cations and ADP, which lie within domains A and B, are well conserved (47-100\%), whereas the effector binding sites, which lie within domain $\mathrm{C}$, are more divergent in some species. In this research, domain A was found to be much more highly conserved (27\%) than domains B (10\%) and $C(3 \%)$. This result is consistent with results obtained for PKs from various species [41]. Interestingly, in the characteristic active site signature of CsPK (IKLIAKIENHEGV), L205 and A207 replace the usual isoleucine (I) and serine $(\mathrm{S})$ found in the rat, mouse and human enzymes. Phylogenetic analysis revealed marked

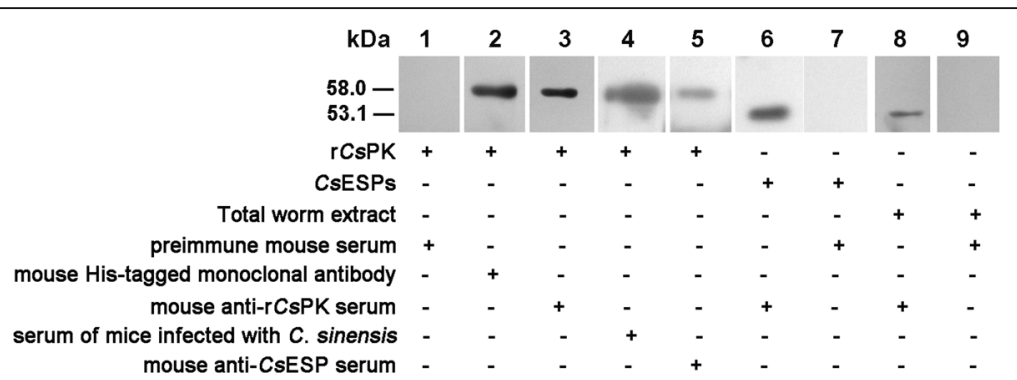

Fig. 4 Western blotting of rCSPK after SDS-PAGE. Blots containing rCSPK were incubated with preimmune mouse serum (Lane 1), a mouse His-tagged monoclonal antibody (Lane 2), mouse anti-rCsPK sera (Lane 3), sera from mice infected with C. sinensis (Lane 4), or mouse anti-CsESPs sera (Lane 5). Blots containing CSESPs were incubated with mouse anti-rCSPK sera (Lane 6) or with preimmune mouse serum (Lane 7). Blots containing total worm extract were incubated with mouse anti-rCsPK sera (Lane 8) or with preimmune mouse serum (Lane 9) 


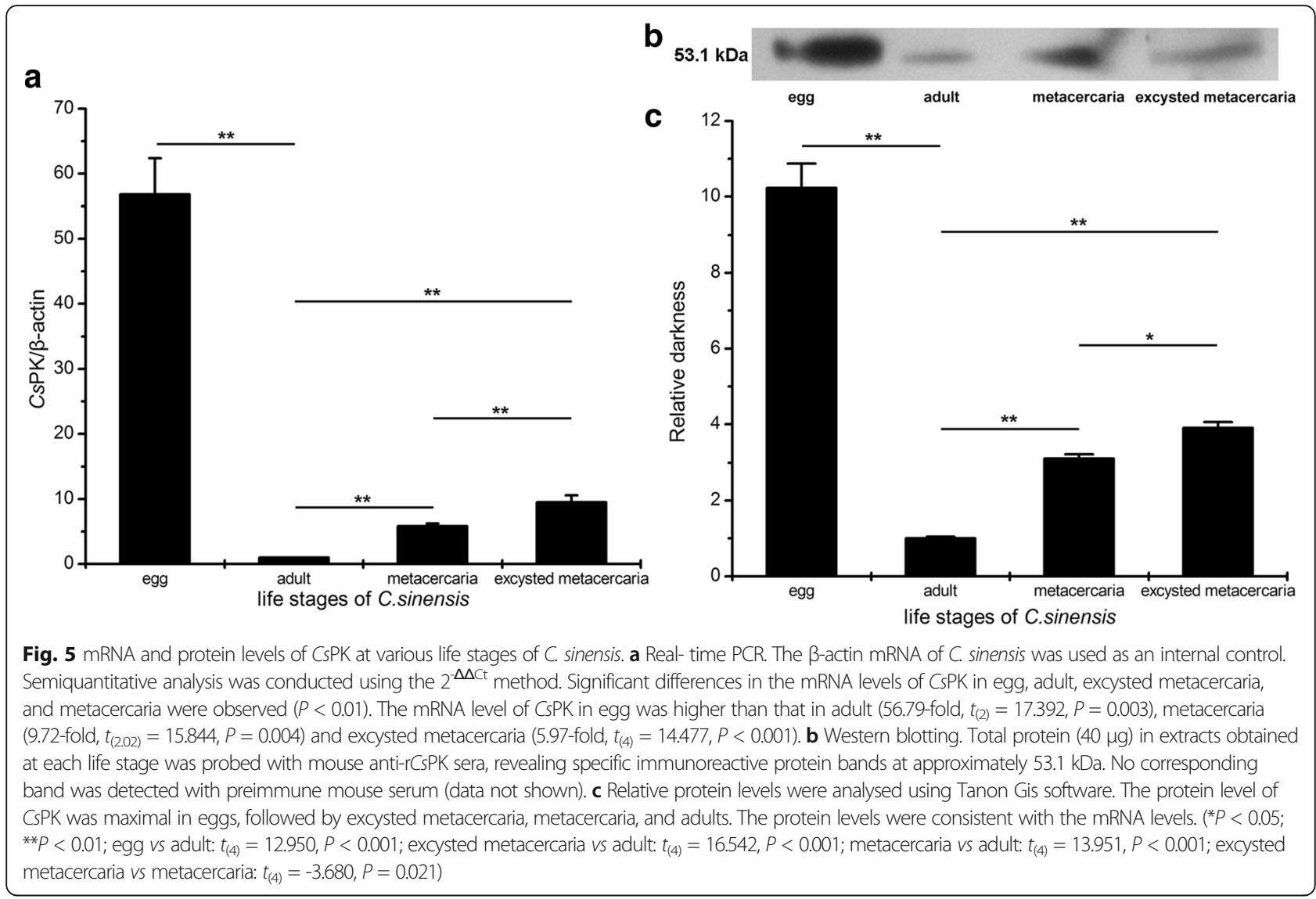

differences between CsPK and PKs from the definitive hosts of $C$. sinensis, cat, rat, human and mouse (Fig. 2).

In contrast to the similarities in the sequence motifs found in the $\mathrm{A}$ and $\mathrm{B}$ domains, the putative sites for effector binding located in domain $\mathrm{C}$, which participate in the allosteric regulation of PKs, are not conserved between parasite and host isoforms. In addition, some sites that are important for effector binding in domain $\mathrm{C}$ and L205 in the characteristic active site signature of the PKs are unique to CsPK (Fig. 1). The findings reported above are particularly interesting with respect to the development of selective and specific inhibitors of parasite PK. Despite the presence of identical catalytic sites and the high sequence conservation (95\%) of the M1-spliced isoform, selective targeting of human PK-M2 that included only the region involved in allosteric regulation was shown to offer a chance of targeting cellular metabolism for the treatment of malignant tumours [40, 42]. This provides proof of principle that PKs might be selectively targeted despite the presence of high degrees of homology among the enzymes from various species [40].

In human PK-M2 and CsPK, the F16BP binding sites, which interact with phosphate moieties [34], are very conservative with different conformations (Additional file 1: Figure S1). In the active sites of CsPK and LmPYK- suramin complexed with glycerol and suramin (an inhibitor of $T$. brucei glycolytic enzymes) [22], the significant residues are highly conserved and display the same conformation (Additional file 1: Figure S1). These observations offer new insight into the mechanism of regulation and the functional features of $C s \mathrm{PK}$ as well as into its potential as a drug target for clonorchiasis.

$\mathrm{rCs} \mathrm{PK}$ reacted with mouse anti-rCsPK sera by Western blotting, revealing its good immunoreactivity. The recognition of $\mathrm{rCs} \mathrm{PK}$ by sera of mice infected with $C$. sinensis hints that $C s \mathrm{PK}$ might be a component of circulating antigens $[43,44]$. Additionally, $\mathrm{rCsPK}$ reacted with mouse anti-CsESPs sera. Furthermore, CsESPs were recognized by mouse anti-rCsPK sera, showing a band of approximately $53.1 \mathrm{kDa}$. These results demonstrate that $C s \mathrm{PK}$ is also a component of ESPs.

As the key glycolytic enzyme, CsPK was widely distributed in the tissues of adult worm, suggesting that CsPK is significant for $C$. sinensis. The locations in which CsPK was found included the pharynx, the intestine, and the tegument, tissues from which ESPs are released [6]. In trematodes, the tegument functions as a dynamic organ that participates in mutual interactions between the parasite and host. Trematode tegument is composed of the syncytial membrane that is highly active in 

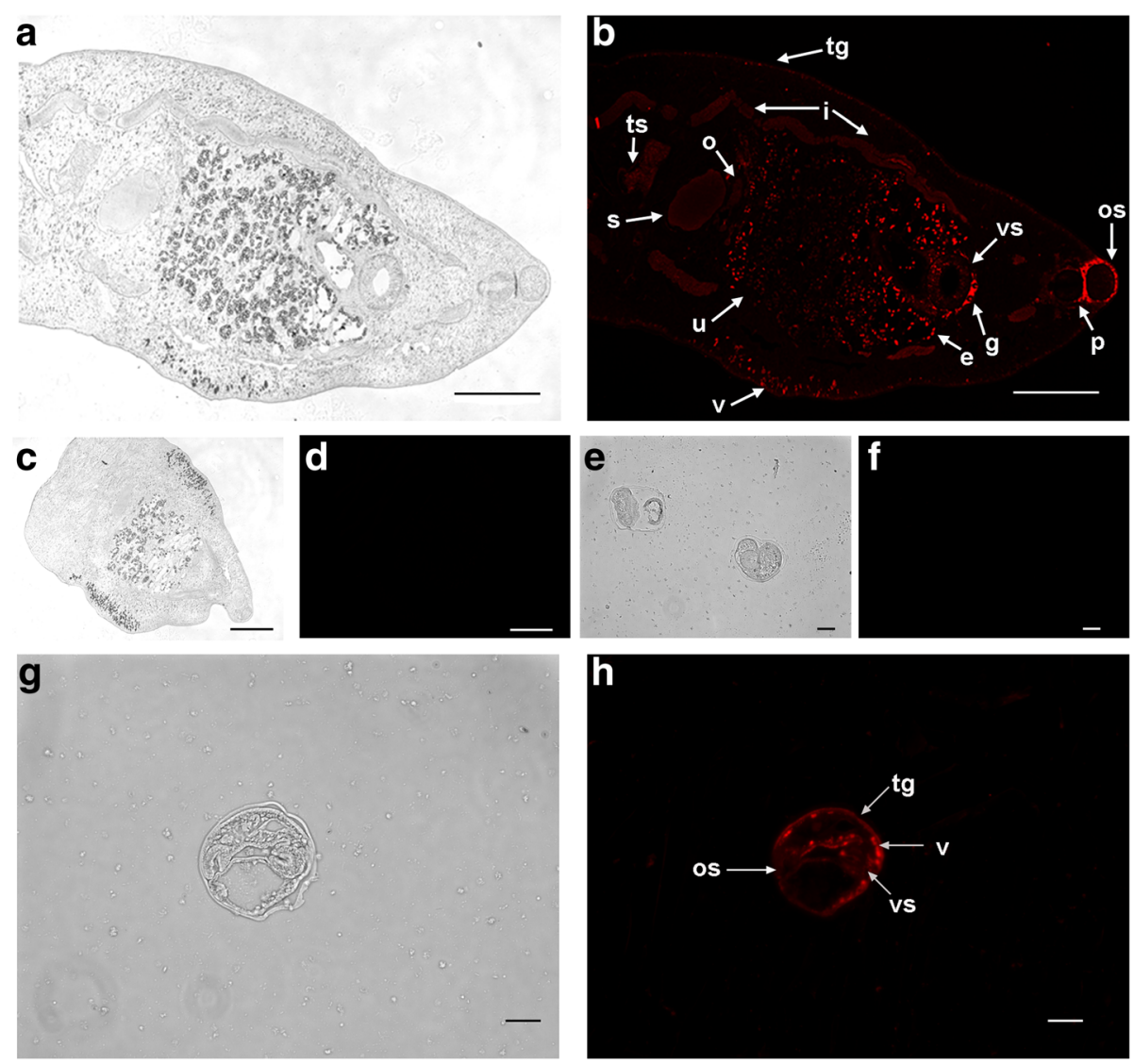

Fig. 6 Immunolocalization of CSPK in C. sinensis. Mouse anti-rCsPK sera and Cy3-conjugated goat anti-mouse lgG were used as the primary and secondary antibodies, respectively. Preimmune mouse serum was used as the primary antibody for the negative controls. Panels (c), (d), (e), and (f) show negative controls. Panels (b), (d), (f), and $\mathbf{h}$ are fluorescence microscopic images; the same areas of the samples photographed under white light are shown in panels (a), (c), (e), and $\mathbf{g}$ with scale-bars. Panel (b), localization of CsPk in adults; panel $\mathbf{h}$, localization of CsPk in metacercariae. Abbreviations: tg, tegument; e, egg; v, vitellarium; os, oral sucker; vs, ventral sucker; g, genital pore; s, seminal receptacle; i, intestine; ts, testicle; u, uterus;o, ovary; p, pharynx. Scale-bars: a-d, $100 \mu \mathrm{m} ; \mathbf{e}-\mathbf{h}, 10 \mu \mathrm{m}$

nutrient uptake. The presence of CsPK in ESPs might result from the shedding and recondition of the tegument [45]. The trematode intestine is a site of nutrient absorption and digestion as well as an important source of ESPs [46]. With the addition of its distribution in tegument, as occurs in fodinichnia, CsPK may be involved in obtaining a sustainable energy supply through the digestion and absorption of glucose from the host.

The localization of CsPK within the musculature, including the pharynx, the oral sucker, and the ventral sucker, may be related to energy demands for adhesion behaviour as well as muscular contraction. Its localization in generative organs, including the ovary, vitellarium, uterus, testis, genital pore, and seminal receptacle, hints that the enzymatic activity of $C s \mathrm{PK}$ in glucose metabolism in the above organs may be needed to satisfy the energy requirements of C. sinensis for growth and reproduction. The vitellarium of the trematode makes a critical contribution to egg production by providing nutrients to the oosperm, providing eggshell material, and related catalytic activities [47].

Bioinformatics analysis indicated that $C s \mathrm{PK}$ contains numerous predicted $\mathrm{T}$ cell as well as $\mathrm{B}$ cell epitopes. The high levels of specific antibodies induced by $\mathrm{r} C s \mathrm{PK}$ may be attributed to its variety of B cell epitopes. It is well known that IgG1 and IgG2a are elicited by Th2 and Th1, respectively. These data suggest that $\mathrm{rCs} \mathrm{PK}$ elicits Th1-biased immunoreaction.

During long-term infection, Th1 to Th2 shift has been shown to occur and to result in long-term persistence of adult worms as well as chronic clonorchiasis [48]. Th1 cells direct protective proinflammatory immune responses [49]. Experiments have shown that the levels of IgG2 and IgG1 antibodies in protected animals are 100-fold and 10fold higher than those of unprotected animals. The protective effect is strongly associated with the avidity, and the titre of IgG2 antibodies elicited [50, 51]. In addition, an effective Th1 immunoreaction is 


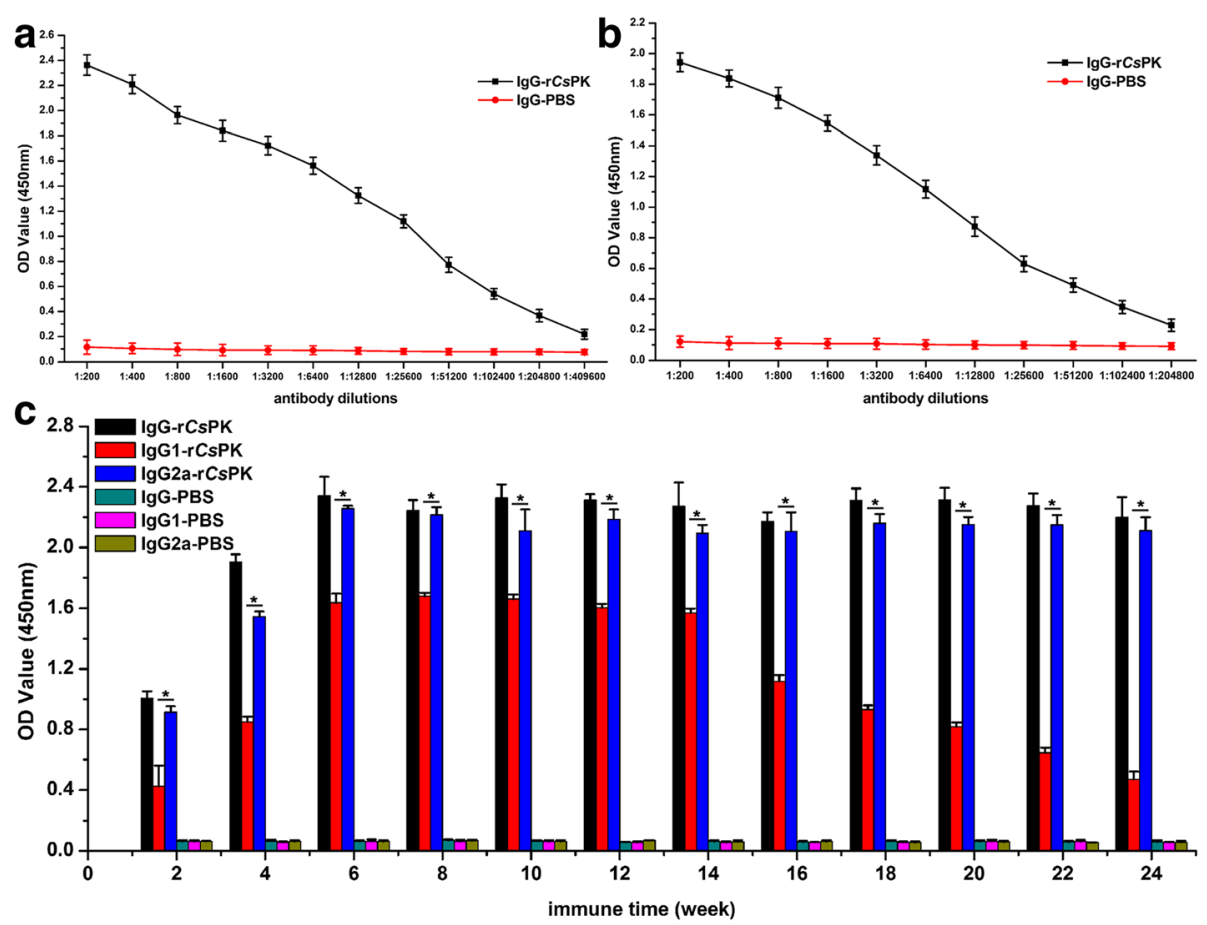

Fig. 7 ELISA determination of antibody titres and isotypes of lgG elicited by rCsPK. Antibody titres of lgG elicited by rCsPK in rats (a) and mice (b). IgG isotypes elicited by $r$ CsPK in rats $(\mathbf{c}) .{ }^{*} P \leqq 0.001 .2$ week: $t_{(6)}=6.886, P<0.001 ; 4$ week: $t_{(6)}=27.959, P<0.001 ; 6$ week: $t_{(6)}=19.829, P<0.001 ; 8$ week: $t_{(6)}=19.278, P<0.001 ; 10$ week: $t_{(6)}=6.264, P=0.001 ; 12$ week: $t_{(6)}=17.319, P<0.001 ; 14$ week: $t_{(6)}=16.977, P<0.001 ; 16$ week: $t_{(6)}=15.057$, $P<0.001 ; 18$ week: $t_{(6)}=37.271, P<0.001 ; 20$ week: $t_{(6)}=48.557, P<0.001 ; 22$ week: $t_{(6)}=40.796, P<0.001 ; 24$ week: $t_{(6)}=32.550, P<0.001$

essential for successful vaccine inoculation against a majority of viral as well as bacterial pathogens [52]. The Th1-biased immune response observed for CsPK revealed its high immunogenicity and might account for its protective efficacy.

Nucleic acid-based, as well as protein-based vaccines for clonorchiasis, have been reported. Some DNA vaccines elicit Th1-biased immune responses, whereas other DNA vaccines and protein vaccines elicit combined Th1/Th2 immune responses [1]. In contrast, rCsPK elicited a Th1-biased immune response. A majority of proteins that are regarded as vaccine candidates are ESPs or tegumental proteins. CsPK was identified as a component of ESPs and was shown to be

Table 1 EPG and worm burden of rats in diverse groups

\begin{tabular}{lll}
\hline Group $(n=8)$ & EPG $^{\mathrm{a}}$ & Worm burden $^{\mathrm{a}}$ \\
\hline PBS & $3983.3 \pm 386.7$ & $25.1 \pm 4.8$ \\
adjuvant & $4075.0 \pm 473.0$ & $24.8 \pm 5.3$ \\
& $\left(t_{(14)}=-0.424, P=0.678\right)$ & $\left(t_{(14)}=0.149, P=0.884\right)$ \\
infection & $3895.8 \pm 424.1$ & $26.1 \pm 5.1$ \\
& $\left(t_{(14)}=0.431, P=0.673\right)$ & $\left(t_{(14)}=-0.407, P=0.690\right)$ \\
rCsPK & $1716.7 \pm 230.3$ & $11.2 \pm 2.5$ \\
& $\left(t_{(11.410)}=14.245, P<0.001\right)$ & $\left(t_{(14)}=7.299, P<0.001\right)$ \\
\hline
\end{tabular}

${ }^{\mathrm{a}}$ Compared with the PBS group deposited on the tegument of adult worms and metacercariae. Rat anti-rCsPK sera inhibited $C$. sinensis adult subsistence in vivo and in vitro. Our results indicate that $C s \mathrm{PK}$ may be a promising candidate for the development of vaccines for clonorchiasis.

\section{Conclusions}

CsPK is a $53.1-\mathrm{kDa}$ homopentamer PK without a characteristic N-terminal domain; it possesses a characteristic active site signature (IKLIAKIENHEGV) and some unique sites. CsPK was widely distributed in the tissues and organs of adult worms, including the oral sucker, ventral sucker, pharynx, genital pore, vitellarium, tegument, intestine, seminal receptacle, testicle, ovary, uterus and in eggs within the uterus. It was shown to be a component of CsESPs. $\mathrm{rCsPK}$ possesses strong immunogenicity as well as immunoreactivity. $\mathrm{rCsPK}$ provoked a Th1-biased immune response. In addition, rat anti-rCsPK sera suppressed $C$. sinensis adult subsistence in vivo and in vitro. This research reveals that CsPK might be a promising vaccine candidate and that it has potential as a drug target against $C$. sinensis infection. The selective development of specific inhibitors against $C s \mathrm{PK}$ and its immune protective mechanisms are worthy of further study. 


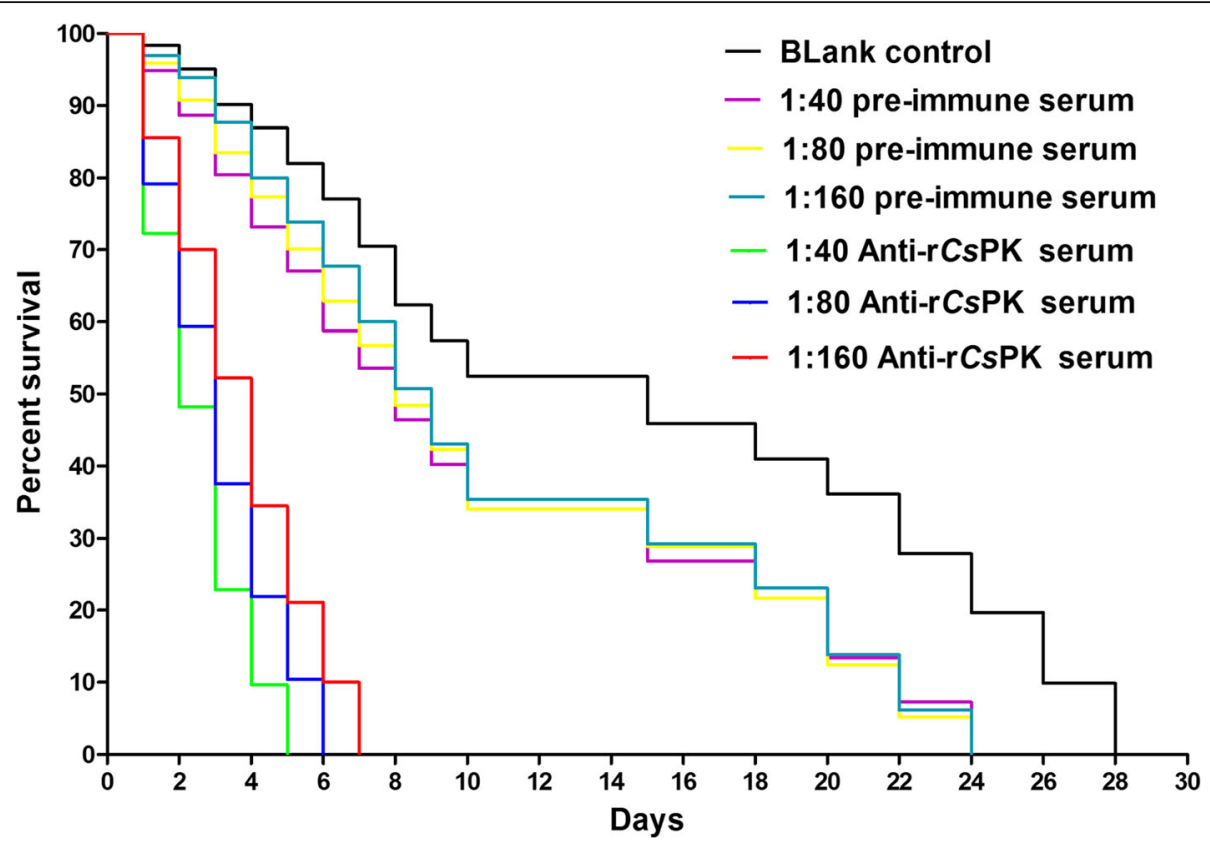

Fig. 8 Rat anti-rCsPK sera inhibits C. sinensis adult subsistence in vitro. The median subsistence times of $C$. sinensis adults in the blank control group, the 1:40 preimmune serum group, the 1:80 preimmune serum group, the 1:160 preimmune serum group, the 1:40 anti-rCsPK serum group, the 1:80 anti-rCSPK serum group, and the 1:160 anti-rCsPK serum group were 15, 8, 8, 9, 2, 3 and 4 days, respectively. No significant difference in the rate of survival of the preimmune serum groups was observed at any serum dilution (1:40 preimmune serum group vs 1:80 preimmune serum group: $X^{2}=0.01289, d f=1, P=0.9096 ; 1: 80$ preimmune serum group vs 1:160 preimmune serum group: $X^{2}=0.09872, d f=1, P=0.7534 ; 1: 40$ preimmune serum group vs 1:160 preimmune serum group: $\left.X^{2}=0.1657, d f=1, P=0.6839\right)$. There were significant differences among the other groups in the rate of survival $\left(1: 40\right.$ anti-rCsPK serum group vs 1:80 anti-rCsPK serum group: $X^{2}=8.058, d f=1, P=0.0045 ; 1: 80$ anti-rCsPK serum group vs 1:160 anti-rCsPK serum group: $X^{2}=8.092, d f=1, P=0.0044$; blank control group vs 1:40 preimmune serum group: $X^{2}=16.15, d f=1$, $P<0.0001$; blank control group vs 1:80 preimmune serum group: $X^{2}=15.54, d f=1, P<0.0001$; blank control group vs 1:160 preimmune serum group: $\left.X^{2}=11.39, d f=1, P=0.0007\right)$

\section{Additional files}

Additional file 1: Figure S1. Putative tertiary modelling of CsPK. K+ and $\mathrm{Mg} 2+$ ions are shown as grey and black spheres, respectively. The N-terminal domain is shown in black. a Ribbon drawing of superposed structure models of CSHK (darker tone) and truncated TgPK1 (lighter tone). The A, B, and C domains of CsHK are shown in blue, red and green, respectively. The catalytic site at the interface of domains $A$ and $B$ and the allosteric site in domain $C$ are highlighted. b Ribbon representation of F16BP (red stick) binding sites of human PK-M2 (lighter tone). S434, S437 (yellow stick), W482 (magenta stick), and R489 (orange stick), which interact with the phosphate moieties, are indicated. The putative corresponding structure of CSPK (darker tone) is shown in panel c. In the active site signature of PK, I267 and S269 (dark red sticks) are replaced by L205 and A207 (blue-violet sticks) in CsHK. Oxalate is indicated as a ball model. $\mathbf{d}$ Ribbon representation of the $\mathrm{K}^{+}$-PK-Mg "oxalate-Mg"ATP complex closed active site (rabbit PK-M1). ATP, oxalate, and significant residues are shown as red, magenta, and yellow sticks, respectively. e Ribbon drawing of the superposition between the A domains of CSPK with the closed rabbit PK-M1 in complex with ATP and oxalate (dark and lighter tones, respectively). The corresponding significant residues of CSPK are shown in orange (stick). $\mathrm{K}^{+}$ and $\mathrm{Mg}^{2+}$ ions, ATP and oxalate, are shown for reference, with their positions derived from a superposition with 1A49. $\mathbf{f}$ Ribbon drawing of superposed structural models of CSPK (darker tone) and LmPYK-suramin (lighter tone) complexed with glycerol (magenta stick) and suramin (an inhibitor of T. brucei glycolytic enzymes, red stick). $\mathbf{g}$ Enlargement of the active site of the LmPYKsuramin structure. Significant residues are coloured yellow (stick). The putative corresponding structure of CSPK is shown in panel $\mathbf{h}$. The corresponding significant residues of CSPK are coloured orange (stick). (TIFF 2964 kb)
Additional file 2: Figure S2. Apparent Mr. of rCsPK. a Determination of the Mr. of rCsPK using 8\% native PAGE according to Fig. 3b. After plotting the $\mathrm{HRm}$ values of the standard markers against their Mrs., we drew a curve, fitted an equation to the curve, and used the equation for calculating the Mr. of rCSPK. $\mathbf{b}$ Elution profile of rCsPK in GFC. $\mathbf{c}$ Mr. of rCsPK as detected with GFC. The calibration curve relating the elution volumes $(\mathrm{Ve})$ and the log $\mathrm{Mr}$. ( $\mathrm{KDa}$ ) of standard marker proteins were obtained with AKTA FPLC using a Sepharose 12 10/300 GL GFC column. Based on the deduced equation, the Mr. of rCSPK was calculated from the obtained Ve of rCSPK. (TIFF $252 \mathrm{~kb}$ )

\section{Abbreviations}

3D: Three-dimensional; BCA: Bicinchoninic acid; BSA: Bovine sera albumin; CSESPS: ESPS of C. sinensis; CSPK: Clonorchis sinensis pyruvate kinase; ELISA: Enzyme-linked immunosorbent assay; EPG: Eggs per gram faeces; ESPs: Excretory/secretory products; HRP: Horseradish peroxidase; Mr.: Molecular mass; MS: Mass spectrometry; ORF: Open reading frame; PBS: Phosphate-buffered saline; PCR: Polymerase chain reaction; PEP: Phosphoenolpyruvate; PK: Pyruvate kinase; SD: Sprague-dawley; Th: T helper cells

\section{Acknowledgements}

We would like to thank Dr Kunhua Hu and Professor Mingtao Li of the Proteomics Centre of Zhongshan School of Medicine, Sun Yat-sen University, for performing the MS analysis in this study. We gratefully thank Yinjuan Wu, Zhipeng Lin, Xinyi Zhou, Lina Zhou, Caiqin Wang, Xiangzhan Kong, Zhuxiu Hou, and Zeli Tang, Department of Parasitology of Zhongshan School of Medicine, Sun Yat-sen University, for excellent technical support in this study. 


\section{Funding}

This work was supported by the national key research and development program of China (No. 2017 YFD0501300 to XY), the Science and Technology Planning Project of Guangdong Province (No. 2016A050502008 to XY), the Young Teachers' Cultivation Project of Basic Scientific Research Service Fee in Colleges and Universities (No.16ykpy06 to TC), the National Natural Science Foundation of China (No. 81772212 to XY). The funders had no role in the study design, data collection and analysis, decision to publish, or preparation of the manuscript.

\section{Availability of data and materials}

DNA sequences and amino acid sequences were obtained from GenBank (https://www.ncbi.nlm.nih.gov/genbank/) and DDBJ (http:// www.ddbj.nig.ac.jp/). Structural files of truncated TgPK1 (PDB: 3GG8), the closed rabbit PK-M1 complexed with ATP and the inhibitor oxalate (PDB: 1A49), human PK-M2 (PDB: 3BJF) and LmPYK-suramin (PDB: 3PP7) were downloaded from the PDB database (http://www.rcsb.org/pdb/home/ home.do). All data generated or analysed during this study are included in this article and its additional files.

\section{Authors' contributions}

Conceived and designed the experiments: TJC, YH, XBY and JX. Performed the experiments: TJC. Analysed the data: TJC, HYJ, HCS and ZZX. Contributed reagents/materials/analysis tools: TJC, PLR, LZ, HMD, MCS, ZYL, ZDW and XRL. Wrote the paper: TJC. YH and XBY gave crucial reviews of this manuscript. All authors read and approved the final manuscript.

\section{Ethics approval and consent to participate}

$\mathrm{SD}$ rats and BALB/C mice were purchased from the animal centre of Sun Yat-sen University and raised carefully according to the National Institutes of Health Guide for the Care and Use of Laboratory Animals. All experimental procedures were authorized by the Animal Care and Use Committee of Sun Yat-sen University (Permit Numbers: SCXK (Guangdong) 2010-0107).

\section{Consent for publication}

Not applicable.

\section{Competing interests}

The authors declare that they have no competing interests.

\section{Publisher's Note}

Springer Nature remains neutral with regard to jurisdictional claims in published maps and institutional affiliations.

\section{Author details}

${ }^{1}$ Department of Parasitology, Zhongshan School of Medicine, Sun Yat-sen University, 74 Zhongshan 2nd Road, Guangzhou, Guangdong 510080, China. ${ }^{2}$ Key Laboratory for Tropical Diseases Control, Sun Yat-sen University, Ministry of Education, Guangzhou, Guangdong 510080, China. ${ }^{3}$ Provincial Engineering Technology Research Centre for Diseases-vectors Control, Guangzhou, Guangdong 510080, China. ${ }^{4}$ Department of Clinical Laboratory, Third Affiliated Hospital, Sun Yat-sen University, Guangzhou, Guangdong 510630, China. ${ }^{5}$ Department of Clinical Laboratory, Second Affiliated Hospital of Guangzhou Medical University, Guangzhou, Guangdong 510260, China.

Received: 15 June 2017 Accepted: 23 October 2017 Published online: 09 November 2017

\section{References}

1. Qian MB, Utzinger J, Keiser J, Zhou XN. Clonorchiasis. Lancet. 2016; 387(10020):800-10.

2. Shin HR, JK O, Masuyer E, Curado MP, Bouvard V, Fang YY, et al. Epidemiology of cholangiocarcinoma: an update focusing on risk factors. Cancer Sci. 2010;101(3):579-85.

3. Qian MB, Chen YD, Yan F. Time to tackle clonorchiasis in China. Inf Dis Poverty. 2013;2(1):4.

4. Bouvard V, Baan R, Straif K, Grosse Y, Secretan B, El Ghissassi F, et al. A review of human carcinogens - part B: biological agents. Lancet Oncol. 2009;10(4):321-2.
5. Tang ZL, Huang Y, Yu XB. Current status and perspectives of Clonorchis sinensis and clonorchiasis: epidemiology, pathogenesis, omics, prevention and control. Inf Dis Poverty. 2016;5(1):71.

6. Wang X, Chen W, Hu F, Deng C, Zhou C, Lv X, et al. Clonorchis sinensis enolase: identification and biochemical characterization of a glycolytic enzyme from excretory/secretory products. Mol Biochem Parasitol. 2011; 177(2):135-42

7. LaCourse EJ, Perally S, Morphew RM, Moxon JV, Prescott M, Dowling DJ, et al. The sigma class glutathione transferase from the liver fluke Fasciola hepatica. Plos Negl Trop Dis. 2012;6(5):e1666.

8. Bae YA, Ahn DW, Lee EG, Kim SH, Cai GB, Kang I, et al. Differential activation of diverse glutathione transferases of Clonorchis sinensis in response to the host bile and oxidative stressors. Plos Negl Trop Dis. 2013;7(5):e2211.

9. Wang $X$, Chen W, Huang Y, Sun J, Men J, Liu H, et al. The draft genome of the carcinogenic human liver fluke Clonorchis sinensis. Genome Biol. 2011; 12(10):R107.

10. Huang Y, Chen W, Wang X, Liu H, Chen Y, et al. The carcinogenic liver fluke, Clonorchis sinensis: new assembly, reannotation and analysis of the genome and characterization of tissue transcriptomes. PLoS One. 2013;8(1):e54732.

11. Kang IK, Lee SH, Seo BS. Study on the (14)C-glucose metabolism by Clonorchis sinensis: paper chromatographic analyses in combination with autoradiography. Korean J Parasitol. 1969;7(3):143-52.

12. Han SS. Autoradiographic studies on the uptake of (14)C-Succinic acid by Clonorchis sinensis. Korean J Parasitol. 1971;9(1):17-24.

13. Hong SJ, Seong KY, Sohn WM, Song KY. Molecular cloning and immunological characterization of phosphoglycerate kinase from Clonorchis sinensis. Mol Biochem Parasitol. 2000;108(2):207-16.

14. Yang G, Jing C, Zhu P, Hu X, Xu J, Wu Z, et al. Molecular cloning and characterization of a novel lactate dehydrogenase gene from Clonorchis sinensis. Parasitol Res. 2006;99(1):55-64.

15. Jamal S, Scaria V. Cheminformatic models based on machine learning for pyruvate kinase inhibitors of Leishmania mexicana. BMC Bioinf. 2013;14:329.

16. Ernest I, Callens M, Uttaro AD, Chevalier N, Opperdoes FR, Muirhead H, et al. Pyruvate kinase of Trypanosoma brucei: overexpression, purification, and functional characterization of wild-type and mutated enzyme. Protein Expr Purif. 1998;13(3):373-82.

17. Maeda T, Saito T, Oguchi Y, Nakazawa M, Takeuchi T, Asai T. Expression and characterization of recombinant pyruvate kinase from Toxoplasma gondii tachyzoites. Parasitol Res. 2003;89(4):259-65.

18. Chan M, Sim TS. Functional analysis, overexpression, and kinetic characterization of pyruvate kinase from Plasmodium falciparum. Biochem Biophys Res Commun. 2005;326(1):188-96.

19. El-Ansary AK, Ahmed SA, Aly SA. Antischistosomal and liver protective effects of Curcuma longa extract in Schistosoma mansoni infected mice. Indian J Exp Biol. 2007;45(9):791-801.

20. Bakszt R, Wernimont A, Allali-Hassani A, Mok MW, Hills T, Hui R, et al. The crystal structure of Toxoplasma gondii Pyruvate Kinase 1. PLoS One. 2010; 5(9):e12736.

21. van Bruggen R, Gualtieri C, lliescu A, Louicharoen Cheepsunthorn C, Mungkalasut $P$, Trape JF, et al. Modulation of malaria phenotypes by pyruvate kinase (PKLR) variants in a Thai population. PLoS One. 2015;10(12):e0144555.

22. Morgan HP, McNae IW, Nowicki MW, Zhong WH, Michels PAM, Auld DS, et al. The trypanocidal drug Suramin and other Trypan blue mimetics are inhibitors of pyruvate kinases and bind to the adenosine site. J Biol Chem. 2011;286(36):31232-40

23. Rigden DJ, Phillips SE, Michels PA, Fothergill-Gilmore LA. The structure of pyruvate kinase from Leishmania mexicana reveals details of the allosteric transition and unusual effector specificity. J Mol Biol. 1999;291(3):615-35.

24. Saito T, Nishi M, Lim Ml, Wu B, Maeda T, Hashimoto $H$, et al. A novel GDP-dependent pyruvate kinase isozyme from Toxoplasma gondii localizes to both the apicoplast and the mitochondrion. J Biol Chem. 2008;283(20):14041-52.

25. Labbe M, Peroval M, Bourdieu C, Girard-Misguich F, Pery P. Eimeria tenella enolase and pyruvate kinase: a likely role in glycolysis and in others functions. Int J Parasitol. 2006;36(14):1443-52.

26. Saavedra E, Olivos A, Encalada R, Moreno-Sanchez R. Entamoeba histolytica: kinetic and molecular evidence of a previously unidentified pyruvate kinase. Exp Parasitol. 2004;106(1-2):11-21.

27. Liang $C, X C H, L v Z Y, Z D$ W, XB Y, et al. Experimental establishment of life cycle of Clonorchis sinensis. Zhongguo ji sheng chong xue yu ji sheng chong bing za zhi. 2009;27(2):148-50. 
28. Chen T, Ning D, Sun H, Li R, Shang M, Li X, et al. Sequence analysis and molecular characterization of Clonorchis sinensis hexokinase, an unusual trimeric 50-kDa glucose-6-phosphate-sensitive allosteric enzyme. PLoS One. 2014;9(9):e107940.

29. Deng C, Sun J, Li X, Wang L, Hu X, Wang X, et al. Molecular identification and characterization of leucine aminopeptidase 2, an excretory-secretory product of Clonorchis sinensis. Mol Biol Rep. 2012;39(10):9817-26.

30. Livak KJ, Schmittgen TD. Analysis of relative gene expression data using real-time quantitative PCR and the 2(-Delta Delta C(T)) method. Methods. 2001;25(4):402-8.

31. Chen T, Yu J, Tang Z, Xie Z, Lin Z, Sun H, et al. Advanced enzymology, expression profile and immune response of Clonorchis sinensis hexokinase show its application potential for prevention and control of clonorchiasis. Plos Negl Trop Dis. 2015;9(3):e0003641.

32. Uddin MH, Li S, Bae YM, Choi MH, Hong ST. Vitro maintenance of Clonorchis sinensis adult worms. Korean J Parasitol. 2012;50(4):309-15.

33. Lee JC. Modulation of allostery of pyruvate kinase by shifting of an ensemble of microstates. Acta Biochim Biophys Sin. 2008;40(7):663-9.

34. Christofk HR, Vander Heiden MG, Wu N, Asara JM, Cantley LC. Pyruvate kinase $\mathrm{M} 2$ is a phosphotyrosine-binding protein. Nature. 2008:452(7184):181-U127.

35. Larsen TM, Benning MM, Rayment I, Reed GH. Structure of the bis(Mg2+)-ATP-oxalate complex of the rabbit muscle pyruvate kinase at 2.1 a resolution: ATP binding over a barrel. Biochemistry. 1998; 37(18):6247-55.

36. Speranza ML, Valentini G, ladarola P, Stoppini M, Malcovati M, Ferri G. Primary structure of three peptides at the catalytic and allosteric sites of the fructose-1,6-bisphosphate-activated pyruvate kinase from Escherichia coli. Biol Chem Hoppe Seyler. 1989;370(3):211-6.

37. Jurica MS, Mesecar A, Heath PJ, Shi W, Nowak T, Stoddard BL. The allosteric regulation of pyruvate kinase by fructose-1,6-bisphosphate. Structure. 1998; 6(2):195-210.

38. Stuart DI, Levine M, Muirhead H, Stammers DK. Crystal structure of cat muscle pyruvate kinase at a resolution of 2.6 a. J Mol Biol. 1979;134(1):109-42.

39. Valentini G, Chiarelli LR, Fortin R, Dolzan M, Galizzi A, Abraham DJ, et al Structure and function of human erythrocyte pyruvate kinase. Molecular basis of nonspherocytic hemolytic anemia. J Biol Chem. 2002;277(26):23807-14.

40. Zoraghi R, See RH, Gong H, Lian T, Swayze R, Finlay BB, et al. Functional analysis, overexpression, and kinetic characterization of pyruvate kinase from methicillin-resistant Staphylococcus aureus. Biochemistry. 2010;49(35):7733-47.

41. Fraunholz MJ, Roos DS. PlasmoDB: exploring genomics and post-genomic data of the malaria parasite, Plasmodium falciparum. Redox Rep. 2003; 8(5):317-20

42. Vander Heiden MG, Christofk HR, Schuman E, Subtelny AO, Sharfi H, Harlow $\mathrm{EE}$, et al. Identification of small molecule inhibitors of pyruvate kinase M2. Biochem Pharmacol. 2010;79(8):1118-24

43. Huang $L$, Lv X, Huang $Y, H u Y$, Yan $H$, Zheng $M$, et al. Identification, sequence analysis, and characterization of serine/threonine protein kinase 17A from Clonorchis sinensis. Parasitol Res. 2014;113(5):1713-23.

44. Zhan JH, Yao JP, Liu W, XC H, ZD W, Zhou XW. Analysis of a nove cathepsin $B$ circulating antigen and its response to drug treatment in Trichinella-infected mice. Parasitol Res. 2013;112(9):3213-22.

45. Gomez-Arreaza A, Acosta H, Quinones W, Concepcion JL, Michels PA, Avilan L. Extracellular functions of glycolytic enzymes of parasites: unpredicted use of ancient proteins. Mol Biochem Parasitol. 2014;193(2):75-81.

46. Lv X, Chen W, Wang X, Li X, Sun J, Deng C, et al. Molecular characterization and expression of a cysteine protease from Clonorchis sinensis and its application for serodiagnosis of clonorchiasis. Parasitol Res. 2012;110(6):2211-9.

47. Cai GB, Bae YA, Kim SH, Sohn WM, Lee YS, Jiang MS, et al. Vitellocyte-specific expression of phospholipid hydroperoxide glutathione peroxidases in Clonorchis sinensis. Int J Parasitol. 2008;38(14):1613-23.

48. Choi YK, Yoon BI, Won YS, Lee $\mathrm{CH}$, Hyun BH, Kim HC, et al. Cytokine responses in mice infected with Clonorchis sinensis. Parasitol Res. 2003 91(2):87-93.

49. Dalton JP, Robinson MW, Mulcahy G, O'Neill SM, Donnelly S. Immunomodulatory molecules of Fasciola hepatica: candidates for both vaccine and immunotherapeutic development. Vet Parasitol. 2013:195(3-4):272-85.
50. Mulcahy G, Dalton JP, Cathepsin L. Proteinases as vaccines against infection with Fasciola hepatica (liver fluke) in ruminants. Res Vet Sci. 2001;70(1):83-6.

51. Mulcahy G, O'Connor F, McGonigle S, Dowd A, Clery DG, Andrews SJ. Correlation of specific antibody titre and avidity with protection in cattle immunized against Fasciola hepatica. Vaccine. 1998;16(9-10):932-9.

52. van Riet E, Hartgers FC, Yazdanbakhsh M. Chronic helminth infections induce immunomodulation: consequences and mechanisms. Immunobiology. 2007;212(6):475-90.

\section{Submit your next manuscript to BioMed Central and we will help you at every step:}

- We accept pre-submission inquiries

- Our selector tool helps you to find the most relevant journal

- We provide round the clock customer support

- Convenient online submission

- Thorough peer review

- Inclusion in PubMed and all major indexing services

- Maximum visibility for your research

Submit your manuscript at www.biomedcentral.com/submit
) Biomed Central 